\title{
Sub-Kilohertz Optical Spectroscopy with a Time Domain Atom Interferometer
}

\author{
F. Ruschewitz, J. L. Peng, H. Hinderthür, N. Schaffrath, K. Sengstock, and W. Ertmer \\ Institut für Quantenoptik der Universität Hannover, Welfengarten 1, D-30167 Hannover, Germany
} (Received 18 November 1997)

\begin{abstract}
We report on the sub-kilohertz optical spectroscopy on the ${ }^{1} S_{0^{-}}{ }^{3} P_{1}$ intercombination transition in magnesium at $457 \mathrm{~nm}$. The spectroscopic signal is probed by a time domain atom interferometer. The realization of this time domain atom interferometer with laser cooled and trapped atoms allows extremely long interaction times and leads to resolutions down to $491 \mathrm{~Hz}$ (FWHM). This corresponds to a high line $Q$ factor of $1.3 \times 10^{12}$. Because of the high accuracy in the determination of the line center, applications with respect to an optical frequency standard are possible. [S0031-9007(98)05752-4]
\end{abstract}

PACS numbers: 03.75.Dg, 39.20.+q, 39.30.+w, 42.50. $-\mathrm{p}$

In recent years the field of atom interferometry [1] has opened up new areas in fundamental and applied atomic physics. This is based mainly on the small wavelength of atomic de Broglie waves leading to the high sensitivity of atomic interferometers. In parallel the operation of atom interferometers has shown that due to the small de Broglie wavelength the proposed high sensitivity demands a setup of highest mechanical stability. Very recently several atom interferometric measurements have reached or even surpassed the precision of conventional "state of the art methods." Prominent examples are the realization of a highly sensitive atom-based Sagnac gyroscope [2], the measurement of the Earth's gravitational acceleration [3], and the precise determination of $\hbar / m_{\text {atom }}$ [4].

The Ramsey-Bordé atom interferometer, in particular, has proven to have excellent perspectives for applications in high resolution spectroscopy [1]. In this type of atom interferometer, the atomic wave is split and the partial waves are reflected and recombined by nearly resonant laser light. The phase shift between the two interfering matter waves depends strongly on the frequency of the splitting laser beam producing the spectroscopic signal. The transfer of the Ramsey-Bordé scheme from the originally proposed beam experiment [5] to a time domain interferometer [6] increases the sensitivity and reduces systematic line shifts by several orders of magnitude.

Different methods for high resolution optical spectroscopy on neutral atoms as well as ions are currently being discussed and evaluated in the context of the next generation of frequency standards and atomic clocks. Especially, cold neutral atoms are also being used in the development of new microwave clocks [7,8]. In this Letter we report for the first time on atom interferometric high resolution measurements of an optical transition with a line $Q$ factor above $10^{12}$ corresponding to a resolved linewidth well below $1 \mathrm{kHz}$. The results presented here should be compared to recent results from the methods of the two-photon spectroscopy [9] and spectroscopy on single trapped ions [10]. In two-photon spectroscopy the ac Stark shift affects the obtainable accuracy, whereas in single-ion spectroscopy the stability is limited by the small particle numbers. The atom interferometric measurement of optical transitions discussed here offers the combination of a high accuracy (potentially better than $2 \times 10^{-15}$ here) as well as a high stability (for an integration time of one second about $2 \times 10^{-13}$ here). The results are also of general importance for prospects in the new area of time domain atom interferometry [3,6,11,12]. Atoms nearly at rest offer both high possible clock accuracies and long interaction times, opening new perspectives for other high precision measurements also.

The basic principle of our time domain interferometer is shown in Fig. 1. Two level atoms initially in the ground state interact with a sequence of two counterpropagating pairs of laser pulses of frequency $\omega_{L}$. Each interaction is nearly resonant with an internal atomic transition with eigenfrequency $\omega_{0}$ and coherently splits the atomic de Broglie wave into two parts labeled by different internal states and different center of mass momenta. According to this entanglement between internal and external atomic states, the two partial waves in the interferometer also

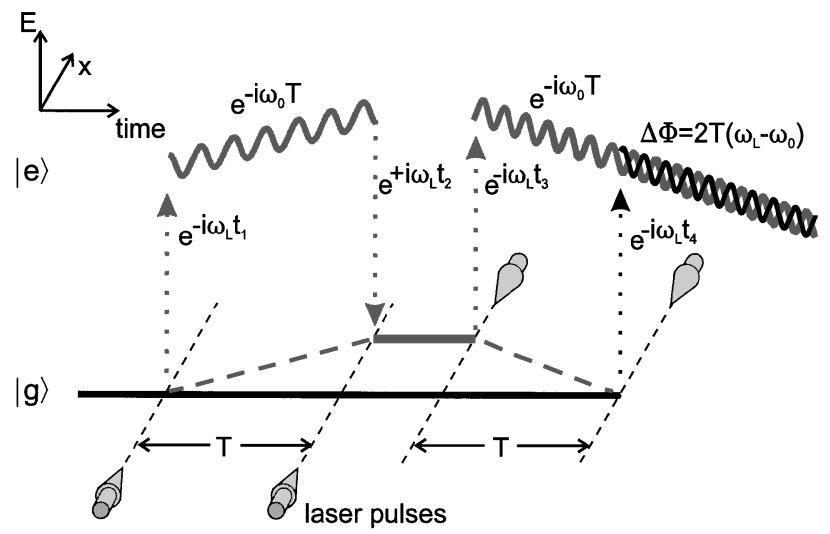

FIG. 1. Scheme of the time domain atom interferometer. Atomic de Broglie waves are split and recombined due to the subsequent interaction with four laser waves. The energy of the ground state is set to zero. During the dark times $T$, the de Broglie wave in the excited state $|e\rangle$ is accumulating the phase $e^{-i \omega_{0} T}$. Each absorbtion (emission) of a photon at the time points $t_{i}$ transfers the additional phase $e^{-i \omega_{L} t_{i}}$ $\left(e^{+i \omega_{L} t_{i}}\right)$. 
separate in space. Therefore, at least four laser pulses are required to provide a spatial overlap between two interfering wave packets at the interferometer exit (Fig. 1). Atomic partial waves in the interferometer accumulate phase differences $e^{-i \omega_{0} T}$ due to their quantum mechanical state evolution during the relevant dark times $T$. Additional phases $e^{\mp i \omega_{L} t_{i}}$ are transferred to the atomic state evolution as a direct consequence of the absorbtion and emission of photons at the time points $t_{i}(i=1, \ldots, 4)$ of the interaction. The overall phase $\Delta \Phi$ of the atomic evolution at one output then appears as the difference of the product of the individual phases accumulated on the two possible paths in the interferometer

$$
\Delta \Phi=2 T\left(\omega_{L}-\omega_{0}\right)+\Phi_{0},
$$

where $T$ denotes the equal dark time periods between the first and second and between the third and fourth laser pulse. $\Phi_{0}$ represents an additional phase offset due to the photon recoil shift [13]. The interference signal is given by the cosine of the phase difference $\Delta \Phi$ and can be easily read out by a state selective detection.

The key feature of the time domain interferometer for its application as a spectroscopic method is the uncomplicated dependency of the interference signal on the laser frequency and the dark time $T$ only. Below, we will discuss the experimental realization of such an interferometer with $\mathrm{Mg}$ atoms.

The singlet and triplet states of alkaline earth elements offer both strong transitions for efficient laser cooling and spin-forbidden intercombination transitions with a long lifetime of the excited states. For magnesium $\left({ }^{24} \mathrm{Mg}\right)$ the closed ${ }^{1} S_{0^{-}}{ }^{1} P_{1}$ transition at $285 \mathrm{~nm}$ $\left(\frac{\Gamma}{2 \pi}=80 \mathrm{MHz}\right)$ allows efficient cooling while the narrow ${ }^{1} S_{0^{-}}{ }^{3} P_{1}$ transition at $457 \mathrm{~nm}\left(\frac{\Gamma}{2 \pi}=31 \mathrm{~Hz}\right.$ [14]) operates the interferometer.

The experimental setup is sketched in Fig. 2. A magneto-optical trap (MOT) is loaded from an effusive thermal magnesium beam. The trapping and cooling light is tuned to the "cooling transition" at $285 \mathrm{~nm}$. The necessary UV radiation is generated by frequency doubling a single-mode dye laser in a beta barium borate (BBO) crystal placed in an external enhancement cavity, the typical UV power being $40 \mathrm{~mW}$. The MOT is operated with $4 \mathrm{~mW}$ in each of the three retroreflected beams of diameter $d_{0}=6 \mathrm{~mm}$. To allow fast switching and active intensity stabilization of the trapping beams, the UV is passed through an acousto-optical modulator (UV-AOM). A spatial filter provides a high quality $\mathrm{TEM}_{00}$ mode. The trap vacuum system is mounted on a freestanding frame to isolate it from vacuum pump vibrations. The base pressure of the trap chamber is $5 \times 10^{-10} \mathrm{mbar}$. Under typical experimental conditions we store about $10^{5}$ atoms. Because of the pure two-level cooling transition, the MOT temperature is close to the Doppler limit $(1.9 \mathrm{mK})$ according to a Gaussian velocity distribution with a width of $\sigma_{v}=0.9 \mathrm{~m} / \mathrm{s}$. The spatial distribution of the stored atoms is Gaussian, too, with a diameter of $\sigma_{d} \approx 500 \mu \mathrm{m}$.

The $457 \mathrm{~nm}$ laser light for the interferometry is generated by a frequency stabilized dye laser spectrometer tuned to the ${ }^{1} S_{0^{-}}{ }^{3} P_{1}$ "clock transition" in magnesium. The linewidth of this laser system is of fundamental importance with respect to the whole experiment. The laser frequency is locked to a high finesse reference cavity $(F=40000)$ by a fast phase modulation technique [15]. The cavity is suspended in vacuum and temperature stabilized. From the error signal of the phase lock loop, we deduce a shot noise limited linewidth of $40 \mathrm{mHz}$ relative to the cavity mode. The "real" spectral width of the locked laser, hence, is determined mainly by the stability of the cavity and details of the setup. An active long term stabilization is provided by locking the laser frequency to an interference fringe of a space domain Ramsey-Bordé interferometer (left part of Fig. 2). This interferometer, consisting of a thermal magnesium atomic beam crossed by two pairs of counterpropagating laser beams, yields

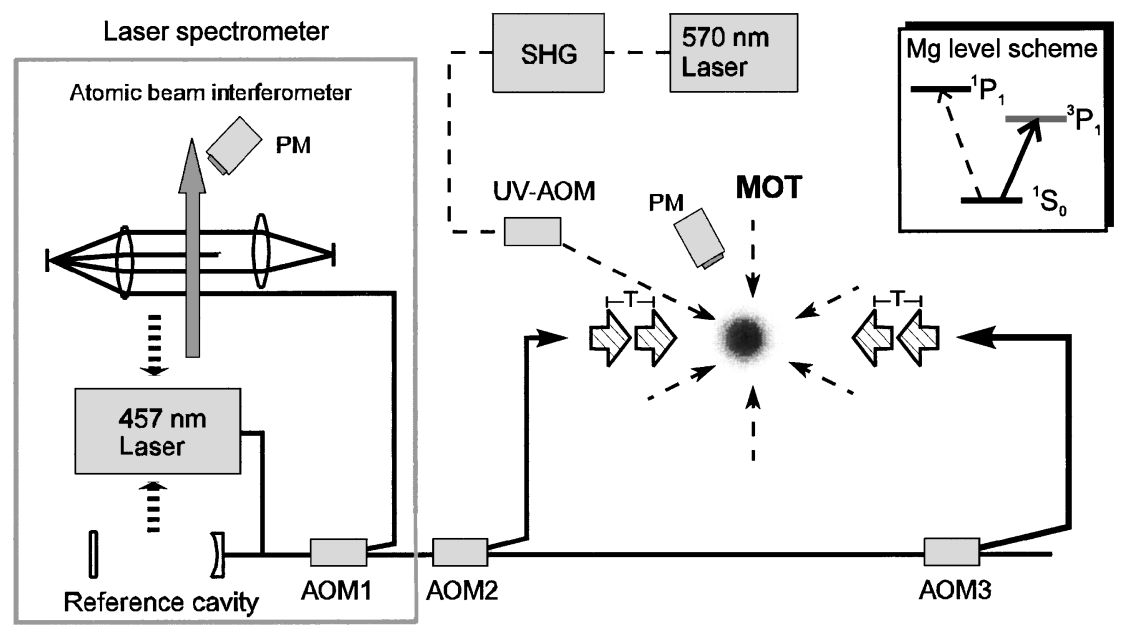

FIG. 2. Sketch of the experimental setup. Dotted arrows on the left represent the frequency locking scheme. The inset on the upper right shows the relevant part of the $\mathrm{Mg}$ level scheme. Details are given in the text. 
resolutions of several kilohertz with a high contrast. Details of this apparatus are given elsewhere [16].

An AOM (AOM1) switches the laser power between the thermal beam apparatus and the trap. Two further AOMs (AOM2,3) form the laser pulses for the time domain interferometer (see below). To obtain a clean spatial mode profile the $457 \mathrm{~nm}$ beam is also passed through a spatial filter. The pointing stability of the different laser beams in parts of the setup is strongly affected by environmental seismic noise. Therefore three active beam pointing stabilizations reduce the geometrical beam instabilities to angular amplitudes of less than $10 \mu \mathrm{rad}$.

Because of the time domain nature of the interferometer scheme, the whole experiment is organized in a time sequence. Each spectroscopic trap cycle is started by loading the trap, then shutting off the UV beams, and switching the magnetic field from a quadrupole shape (as needed for the MOT) to a homogeneous offset field. This offset field shifts the $m= \pm 1$ levels of the metastable ${ }^{3} P_{1}$ state out of resonance. About $50 \mu$ s later, the freely expanding cloud of cold ground state atoms is irradiated with the sequence of four $457 \mathrm{~nm}$ laser pulses which constitute the atom interferometer. Each pulse has a width of $6 \mu$ s representing a $\pi / 2$ pulse. For different scans the dark time $T$ (see Fig. 1) has been varied, resulting in different interference periodicities according to Eq. (1). After the last pulse, the trap is switched on again which completes a spectroscopic cycle.

As a consequence of the interference process a number of atoms are excited to the metastable ${ }^{3} P_{1}$ state corresponding to the excited state exit port of the interferometer. As the atoms in this state will not be recaptured on the fast $285 \mathrm{~nm}$ transition this leads to a trap loss mechanism which depends on the population of the corresponding interferometer exit.

Thus, monitoring the trap fluorescence, we take advantage of the high quantum efficiency of the strong cooling transition to read out the interferometer exit signal which is encoded in the excitation probability on the weak clock transition. From single ion spectroscopy a similar method is known as "electron shelving" [17].

According to the narrow spectral width of the ${ }^{3} P_{1}$ excitation, only a small velocity class takes part in the interferometry process. We repeat the spectroscopy cycles at a rate of typically $50 \mathrm{~Hz}$, so that for a trap lifetime $\tau_{0}$ of one second each atom has about 50 chances to jump to the metastable state before leaving the trap for other reasons. This periodic scheme results in a further amplification of the trap loss, leading to the high contrast detection of the small excitation probabilities on the weak clock transition.

Figure 3 shows representative data for time domain interference signals as a function of the laser frequency for different dark times $T$ between the interferometry pulses. The lines represent cosine fits. As is typical for interferometry with two waves the interference pattern resembles a cosine shape. Note that the observed signals span a wide frequency range in contrast to interferences

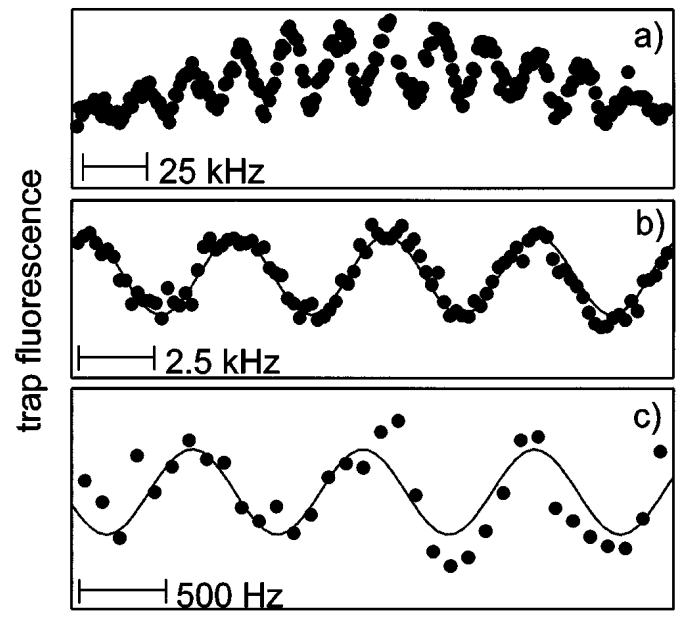

FIG. 3. Time domain interference signals detected as a function of the laser frequency. Scans for three different resolutions corresponding to different dark times $T$ are shown: (a) $T=12.6 \mu \mathrm{s}$; (b) $T=100 \mu \mathrm{s}$; (c) $T=509 \mu \mathrm{s}$. The integration time per data point is for (a) and (b) $1.1 \mathrm{~s}$, for (c) $2.2 \mathrm{~s}$.

obtained from thermal beam ensembles, where the small coherence length reduces the visibility to only a few fringes. The contrast envelope which appears in Fig. 3(a) is due to the finite spectral width of the laser excitation. The periodicity of the signals decreases with increasing $T$.

For the realization of an optical frequency standard we have to consider the frequency stability of a laser locked to the minimum of a central interference fringe. The calculated shot-noise limited fractional frequency stability for the observed signals of Fig. 3 is some $10^{-13}$ [for example, $\frac{\Delta \nu}{\nu}=2.7 \times 10^{-13}$ for Fig. 3(b)]. This stability is proportional to the inverse of the signal-to-noise $(\mathrm{S} / \mathrm{N})$ ratio and the line $Q$ factor. To verify that the noise in our signals is white noise we measured the relative frequency fluctuations of the atomic beam signal relative to the central fringe of the trap signal [indicated in Fig. 3(b)] and computed a two-sample (Allan) variance. For integration times $\tau$ up to several hundred seconds, this Allan variance shows a linear dependence on $\tau^{-1 / 2}$ indicating white noise.

In estimating the accuracy we found that most types of systematic line shifts are significantly reduced in the time domain interference signal of the trapped atoms compared to atomic beam signals. The overall projected accuracy is already as high as $\frac{\Delta \nu}{\nu}=2 \times 10^{-15}[18]$.

An example of high spectroscopic resolutions is given in Fig. 3(c). Here the fringes show the extremely narrow FWHM linewidth of $491 \mathrm{~Hz}$. The $\mathrm{S} / \mathrm{N}$ ratio for the data is 3.3. For the given clock transition frequency of $\nu=655 \mathrm{THz}$, we calculate a line $Q$ factor of $1.3 \times 10^{12}$. To our knowledge this is the highest line $Q$ factor which has been observed for neutral atoms so far.

The $\mathrm{S} / \mathrm{N}$ ratio of the interference signals strongly decreases with increasing resolution. For a given constant noise this is due to a systematic loss of interference 


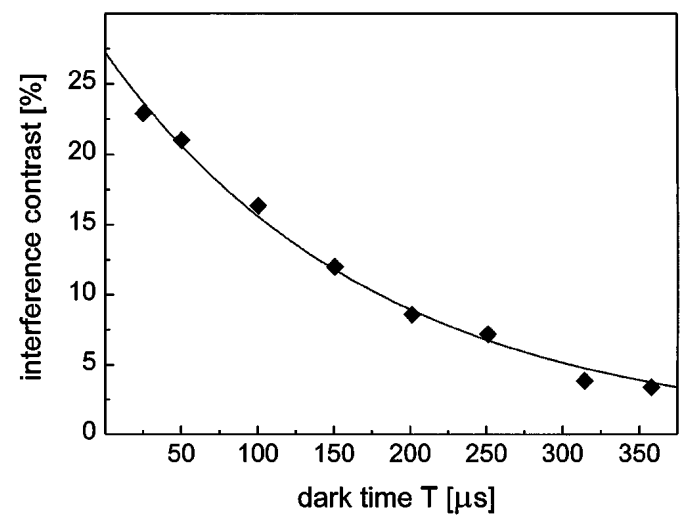

FIG. 4. Contrast of the interference signals as a function of the dark time $T$. The exponential loss of contrast is attributed mainly to the finite linewidth of the laser excitation. The fit curve yields a linewidth of $(846 \pm 42) \mathrm{Hz}$. (Additional effects, mentioned in the text, are taken into account.)

contrast. In the following we will give a brief discussion of the various effects which influence the signal contrast.

Figure 4 shows the interference contrast as a function of the dark time $T$ extracted from a large number of data sets. The contrast significantly decreases as $T$ increases. Especially, the effective linewidth $\delta \nu$ of the laser excitation leads to a strong exponential decay of the interference contrast function $C(T)$ [18],

$$
C(T) \propto e^{-2 \pi \delta \nu T} .
$$

Effects which give rise to additional slight losses of contrast (calculated for $T=250 \mu \mathrm{s}$ ) are the variation in the excitation probability $(3.8 \%)$ and the first order Doppler effect (1\%) due to the Gaussian intensity profile of the spectroscopy laser beams, the spontaneous decay of the ${ }^{3} P_{1}$ state $(0.3 \%)$, and the first order Doppler effect due to misalignment of the counterpropagating spectroscopy laser beams $(0.2 \%)$. Accordingly the influence of the Sagnac effect, gravity, black body radiation, and the second order Doppler effect to the contrast is below $0.001 \%$.

Taking the main effects into account, we compute a real laser linewidth of $(846 \pm 42) \mathrm{Hz}$ from the data. For typical short integration times of about one second per data point, this value is attributed to frequency fluctuations of the spectrometer cavity.

These estimations show that the presented method of high resolution frequency discrimination in a time domain atom interferometer is not yet limited by any fundamental effects. At present, the obtainable resolutions depend mainly on the linewidth of our laser spectrometer. Considerable improvements of the spectrometer cavity and the fast phase lock loop are in progress. This should improve the resolution by an order of magnitude corresponding to resolvable linewidths in the regime of the natural atomic state linewidth of the ${ }^{3} P_{1}$ state of $31 \mathrm{~Hz}$.

Recently, a group from the Physikalisch-Technische Bundesanstalt in Braunschweig also obtained resolutions below $500 \mathrm{~Hz}$ on the intercombination transition in calcium at $657 \mathrm{~nm}$ with a natural linewidth of $410 \mathrm{~Hz}$ [20].
An advantage of this interferometric method compared to other recent experiments is the high accuracy in the determination of the exact line center as well as the high stability of the signals. Therefore, high resolution spectroscopy with a time domain atom interferometer suggests the application as a frequency standard. For this purpose, the direct measurement of the transition frequency compared to a primary frequency standard with a so-called frequency chain [19] is considered.

We appreciate the experimental contributions of J. Lohe. This work was supported by the Deutsche Forschungsgemeinschaft under SFB 407. J. L.P. acknowledges support from the DAAD.

[1] See, e.g., Atom Interferometry, edited by P. Berman (Academic Press, New York, 1997).

[2] T.L. Gustavson, P. Bouyer, and M. A. Kasevich, Phys. Rev. Lett. 78, 2046 (1997).

[3] M. Kasevich and S. Chu, Appl. Phys. B 54, 321 (1992).

[4] D. S. Weiss, B. C. Young, and S. Chu, Appl. Phys. B 59, 217 (1994).

[5] C. J. Bordè, Phys. Lett. A 140, 10 (1989).

[6] K. Sengstock, U. Sterr, G. Hennig, D. Bettermann, J. H. Müller, and W. Ertmer, Opt. Commun. 103, 73 (1993).

[7] A. Clairon, P. Laurent, G. Santarelli, S. Ghezali, S. N. Lea, and M. Bahoura, IEEE Trans. Instrum. Meas. 44, 128 (1995).

[8] K. Gibble and S. Chu, Phys. Rev. Lett. 70, 1771 (1993).

[9] F. Schmidt-Kaler, D. Leibfried, S. Seel, C. Zimmermann, W. König, M. Weitz, and T. W. Hänsch, Phys. Rev. A 51, 2789 (1995).

[10] J. C. Bergquist, W. M. Itano, and D. J. Wineland, in Frontiers in Laser Spectroscopy, edited by T. W. Hänsch and M. Inguscio (North-Holland, Amsterdam, 1994), p. 359.

[11] S. B. Cahn, A. Kumarakrishnan, U. Shim, T. Sleator, P. R. Berman, and B. Dubetsky, Phys. Rev. Lett. 79, 784 (1997).

[12] P. Szriftgiser, D. Guéry-Odelin, M. Arndt, and J. Dalibard, Phys. Rev. Lett. 77, 4 (1996).

[13] C. J. Bordé, C. Salomon, S. Avrillier, A. van Lerberghe, C. Bréant, D. Bassi, and G. Scoles, Phys. Rev. A 30, 1836 (1984).

[14] A. Godone and C. Novero, Phys. Rev. A 45, 1717 (1992).

[15] R. W. P. Drever, J.L. Hall, F. V. Kowalsky, J. Hough, G. M. Ford, H. Munley, and H. Ward, Appl. Phys. B 31, 97 (1983).

[16] H. Hinderthür, A. Pautz, F. Ruschewitz, K. Sengstock, and W. Ertmer, Phys. Rev. A (to be published).

[17] H. G. Dehmelt, Bull. Am. Phys. Soc. 20, 60 (1975).

[18] K. Sengstock, U. Sterr, J.H. Müller, V. Rieger, D. Bettermann, and W. Ertmer, Appl. Phys. B 59, 99 (1994).

[19] H. Schnatz, B. Lipphardt, J. Helmcke, F. Riehle, and G. Zinner, Phys. Rev. Lett. 76, 18 (1996).

[20] G. Zinner, Ph.D. thesis, Physikalisch-Technische Bundesanstalt, Braunschweig, 1997. 